\title{
Sistema SCADA adaptado para el control de la alimentación en animales de laboratorio
}

\author{
Julia María Samos Juárez \\ Directora del animalario de la Facultad de Medicina de Albacete \\ de la Universidad de Castilla-La Mancha \\ JuliaMaria.Samos@uclm.es \\ Jorge Juan Rosillo Olmos \\ CTO de Konery \\ jorgejuan@konery.com \\ Antonio Ruiz Canales \\ Profesor titular de universidad del Departamento de Ingeniería \\ de la Universidad Miguel Hernández de Elche \\ acanales@umh.es
}

\section{Extracto}

Las instalaciones dedicadas al alojamiento de animales de laboratorio son cada vez más complejas. No solo deben garantizar las necesidades básicas y el bienestar de los animales alojados, sino que también deben permitir la realización de la variedad de procedimientos experimentales que tienen lugar en ellas.

Debido a la dificultad de implementación y al alto coste de los sistemas automáticos de alimentación, el uso de los mismos se ha limitado prácticamente a grandes instalaciones, siendo manual el suministro de dietas en la mayoría de centros. No obstante, debido al incremento de procedimientos experimentales que incluyen algún tipo de restricción alimentaria y, principalmente, a las ventajas en roedores de la alimentación restringida frente a la alimentación ad libitum (AL) en estudios a largo plazo, el grado de sofisticación de los sistemas automáticos de alimentación para animales de experimentación es superior a los de otras especies.

Dado el estado de crecimiento de este sector, surge la idea de desarrollar e implementar un dispensador de dieta de bajo coste para roedores de experimentación. En este trabajo se plantea el desarrollo de un prototipo, a partir de un sistema SCADA (supervisory control and data acquisition). El usuario accederá al control del equipo a través de una interfaz de fácil instalación y uso. El SCADA permitirá la dosificación del alimento según un programa-horario establecido y el registro de la ingesta en tiempo real. Esta solución de bajo coste establecerá una propuesta competitiva y de uso extensivo en el mercado.

Palabras clave: ratas; sistema de bajo coste; control de alimentación; Arduino®; inalámbrico.

Fecha de entrada: 22-07-2019 / Fecha de revisión: 01-12-2019 / Fecha de aceptación: 14-01-2020

Cómo citar: Samos Juárez, J. M. ${ }^{a}$, Rosillo Olmos, J. J. y Ruiz Canales, A. (2020). Sistema SCADA adaptado para el control de la alimentación en animales de laboratorio. Tecnología, Ciencia y Educación, $16,143-165$. 


\title{
SCADA system adapted for feeding control in laboratory animals
}

\author{
Julia María Samos Juárez \\ Jorge Juan Rosillo OImos \\ Antonio Ruiz Canales
}

\section{Abstract}

Animal facilities are becoming increasingly complex, they must ensure the basic needs and animal welfare, and moreover they should allow the implementation of the variety of experimental procedures that take place in them. Due to the difficulty of implementation and the high cost of the automatic feeding systems, the use of these systems has been practically limited to large facilities, being the provision of diets manual in most centres. However, due to the increase in experimental procedures involving some type of food restriction and mainly to the advantages of restricted feeding versus ad libitum (AL) feeding in long-term rodent studies, the sophistication of automatic feeding systems in experimental animals is higher than in another species.

The idea of developing and implementing a low-cost diet dispenser for experimental rodents comes up given the huge potential for growth in this sector. Following this concept, we propose in this article the development of a prototype, based on a SCADA (supervisory control and data acquisition) system. The user will access to the control of the equipment through an interface easy to install and to use. The SCADA will allow the dosage of the food according to an established schedule and the recording of the intake in real time. This low-cost solution will establish a competitive and widely used proposal on the market.

Keywords: rats; low cost systems; feed control; Arduino®; wireless.

Citation: Samos Juárez, J. M. ${ }^{a}$, Rosillo Olmos, J. J. y Ruiz Canales, A. (2020). SCADA system adapted for feeding control in laboratory animals. Tecnología, Ciencia y Educación, 16, 143-165. 


\section{Sumario}

1. Introducción

2. Material y métodos

\subsection{Sensores}

2.2. Software y comunicaciones

2.3. Descripción del software

2.4. Software programador de dietas de roedores de laboratorio

$$
\text { 2.4.1. Inicio del software }
$$

2.4.2. Estado del sistema

2.4.3. Configuración

2.4.4. Programador

2.4.5. Salidas formato Excel

2.4.6. Salidas gráficas en tiempo real

3. Resultados y discusión

4. Limitaciones del software

5. Conclusiones

Referencias bibliográficas

Nota: este trabajo se enmarca dentro de la Convocatoria de Ayudas a Proyectos de 1+D+i 2017 de la Fundación Hergar (categoría: Aplicación de las TIC a la educación de adultos y a las ciencias de la salud). 


\section{Introducción}

En animales de abasto, la distribución de la alimentación de manera automática es un hecho. La mejora y evolución de estos sistemas ha sido continua, existiendo en el mercado diferentes dispositivos que se adaptan a la especie, a la edad y a los diferentes sistemas de alojamiento. En los últimos años, y también en el sector ganadero, se han desarrollado sistemas de alimentación inteligente en los que se proporciona a los animales los nutrientes que necesitan en función del estado productivo y/o reproductivo en el que se encuentren. Esto permite un control de los costes de la alimentación y una flexibilización de las rutinas de trabajo, reduciendo la generación de desechos y el consumo energético.

\begin{abstract}
En el caso de los animales de laboratorio, aunque el número de dispositivos comerciales es menor, también se han generado grandes avances, tanto en sistemas de alojamiento como en sistemas automáticos de distribución de dieta. Esto ha facilitado la monitorización durante largos periodos de tiempo de diferentes parámetros fisiológicos y de comportamiento, de condiciones corporales e incluso de actividad a nivel cerebral
\end{abstract}

En el caso de los animales de laboratorio, aunque el número de dispositivos comerciales es menor, también se han generado grandes avances, tanto en sistemas de alojamiento como en sistemas automáticos de distribución de dieta. Esto ha facilitado la monitorización durante largos periodos de tiempo de diferentes parámetros fisiológicos y de comportamiento, de condiciones corporales e incluso de actividad cerebral (Bolaños, Le Due y Murphy, 2017; Goulding et al., 2008; Naoto, 2015; Ulman, Compton y Kochanek, 2008). Además, al disminuir la interacción con el operador, se minimizan las alteraciones en parámetros fisiológicos y de comportamiento ocasionados por la manipulación (Balcombe, Barnard y Sandusky, 2004).

En la mayoría de procedimientos experimentales realizados con animales, el control del peso es determinante. Este debe ser observado cuidadosamente, ya que es uno de los parámetros más importantes a la hora de evaluar la salud y el bienestar animal y de establecer criterios de punto final. Para el control del peso se han desarrollado sistemas automáticos de pesaje y seguimiento en roedores. Este tipo de sistemas

\author{
En la mayoría de procedimientos \\ experimentales realizados con \\ animales, el control del peso \\ es determinante, ya que resulta uno \\ de los parámetros más importantes \\ a la hora de evaluar la salud y el \\ bienestar animal
}


permite a los investigadores monitorizar la fluctuación del peso corporal durante el periodo de tiempo elegido, sin interacción humana, obtener datos precisos (Murphy et al., 2016; Scott, Brody y Tank, 2013) e incluso valorar el comportamiento dentro de las jaulas (Bains et al., 2016; Richardson, 2015). No obstante, para roedores, es difícil encontrar en el mercado un sistema de pesado integrado en la jaula y que permita mantener a los animales en grupo.

Otro tema muy importante es el relativo al régimen de administración de dietas en animales de laboratorio. En roedores, normalmente es AL. Aunque esta práctica de alimentación tiene algunos inconvenientes descritos en la literatura (Keenan et al., 1996), sigue siendo el régimen alimenticio más extendido en todo el mundo. En este contexto, el término «restricción de dieta» (diet restriction [DR]) se usa para describir cualquier método de restricción de ingesta de comida dirigida a la disminución de la ingesta calórica comparada con los animales alimentados AL.

En contraste a los regímenes con restricción, la alimentación AL se ha asociado tanto a alta morbilidad como a una reducción en la esperanza de vida (Keenan et al., 1996; Masoro, 2005). Los beneficios de la DR se atribuyen a la disminución en la ingesta calórica (Masoro, 2005). Específicamente, el régimen en $\mathrm{DR}$ reduce la incidencia de neoplasias (Duffy, Lewis, Mayhugh, Trotter, Thorn

En contraste a los regímenes con restricción, la alimentación AL se ha asociado tanto a alta morbilidad como a una reducción en la esperanza de vida de los animales et al., 2004; Hubert, Laroque, Gillet y Keenan, 2000; Masoro, 2005), de alteraciones endocrinas (Keenan y Soper, 1995; Keenan et al., 1996), cambios degenerativos del riñón (Duffy, Lewis, Mayhugh, Trotter, Latendresse et al., 2004; Hubert et al., 2000;) y de otras causas comunes de morbilidad (Duffy, Lewis, Mayhugh, Trotter, Thorn et al., 2004; Hubert et al., 2000) en roedores de laboratorio. Además, al estar más sanos, los animales en DR se enfrentan mejor a situaciones de estrés; por ejemplo, se recuperan mejor de procedimientos quirúrgicos y presentan menor respuesta inflamatoria (Keenan et al., 1996; Masoro, 1995). En animales jóvenes los cambios más evidentes se observan antes en los parámetros fisiológicos que en las pautas de morbilidad y mortalidad. En estos casos se ha demostrado que la DR disminuye el contaje de leucocitos y los números de neutrófilos y linfocitos (Hubert et al., 2000; Keenan et al., 1998; Roe et al., 1995), mientras que incrementa el número de eritrocitos, hematocritos y hemoglobina (Pickering y Pickering, 1984; Roe et al., 1995). La DR también modifica el metabolismo de los roedores (Turturro et al., 1993), resultando una disminución total en suero de colesterol HDL, triglicéridos y lípidos (Hubert et al., 2000; Keenan et al., 1998; Turturro et al., 1993). Además de en el metabolismo de las grasas, la DR tiene impacto en otros parámetros químicos clínicos. Se ha visto que disminuye la proteína total en suero y la cantidad de albúmina y globulina en plasma (Hubert et al., 2000; Roe et al., 1995; Snyder y Towne, 1989), aunque la extensión de esos efectos depende del método y de la severidad del régimen DR aplicado. La alimentación restringida también puede conducir a una reducción de la variabilidad interindividual. Las grandes variaciones en el peso, la supervivencia y la incidencia de tumores son problemas bien conocidos, asociados con animales alimentados AL (Keenan et al., 
1996), donde el peso corporal tiene una gran influencia en la variabilidad de aparición de tumores (Turturro et al., 1996). La DR podría ayudar a resolver estos problemas. Además, la implementación de protocolos de restricción alimentaria puede ser una manera de incentivar la participación de los animales en experimentos de comportamiento.

En el campo del bienestar animal hemos observado que el uso de un régimen alimenticio con DR conduce a la reducción, según el método de las tres erres (3 R) (Russell and Burch, 1959), en el número de animales usados en estudios a largo plazo. En este tipo de estudios, si los roedores se alimentan $\mathrm{AL}$, se requerirá la inclusión de un mayor número de animales para compensar las pérdidas por morbilidad y mortalidad. Esto es especialmente importante en estudios para la evaluación de seguridad que pueden durar hasta 24 meses. El uso de la DR en este tipo de estudios implicaría un descenso anual en el número de animales utilizados (Hubert et al., 2000). Además, se ha demostrado que la DR reduce la varianza de los datos en varios parámetros (Carney et al., 2004; Leakey, Seng y Allaben, 2003). La implementación de una DR moderada (75-80 \% de AL) podría llegar a ser una poderosa herramienta de reducción (Ritskes-Hoitinga y Savenije, 2008).

Los motivos por los que la DR no se usa de manera rutinaria son varios. Uno de los principales es la necesidad de alojar a los roedores de manera individualizada. En roedores esto es un gran inconveniente, ya que, al ser animales gregarios, por razones de bienestar animal deben alojarse en grupos. Por otra parte, restringir la cantidad de comida ofrecida a un grupo de roedores puede, debido a la jerarquía social, dar lugar a un acceso desigual a los alimentos. Por lo tanto, el grado de restricción puede ser diferente entre los individuos alojados en la misma jaula, y esto podría contrarrestar uno de los objetivos deseados, es decir, disminución de la varianza. Se ha desarrollado un dispositivo capaz de controlar la ingesta de alimentos de manera individualizada en ratas estabuladas en grupo (Laboratory Feeding Systems [Dinamarca]), pero, por el momento, parece ser demasiado caro y complicado para el uso rutinario en roedores de laboratorio. Otro factor que hay que considerar es el ritmo circadiano de los animales. Cuando se alimentan con AL, las ratas comen principalmente durante la fase nocturna en varias franjas horarias (Spiteri, 1982). A menudo, la DR se pone en práctica dando a las ratas, alojadas individualmente, su ración diaria de alimento una vez al día durante la fase de luz, simplemente por razones prácticas. Los animales consumen su ración diaria rápidamente, lo que tiene un profundo efecto en muchos parámetros fisiológicos regulados por el ritmo circadiano (Chacón et al., 2005; Feuers, 1991). Se han desarrollado métodos de DR que permiten a las ratas estar alojadas en un grupo y, con una alta probabilidad, mantener su ritmo circadiano (Kemppinen, Meller, Mauranen, Kohila y Nevalainen, 2008). Este método es un simple dispositivo llamado «diet board». Este estudio fue diseñado para evaluar si la DR puede modificar determinados parámetros fisiológicos 
y clínicos, y su influencia en el aumento de peso y el crecimiento de los animales. El sistema consiste en dos tablas de madera donde se encajan los pellets de la dieta. Los animales tienen acceso a la comida todo el tiempo, pero deben roer la madera antes de alcanzar los gránulos, por lo que la dieta en sí podría proporcionar un nivel de dificultad ideal para obtener comida. Esto daría como resultado que los animales solo coman la cantidad que realmente necesitan, con lo que se obtendrían todas las ventajas asociadas a la DR.

Llegados a este punto nos planteamos la posibilidad de diseñar un dispositivo que controle el acceso a la dieta y que cumpla con la mayoría de los argumentos anteriores. Nuestro grupo cuenta con una amplia experiencia en el diseño de sistemas SCADA en agricultura para el control del riego de manera automática (Molina-Martínez et al., 2014), permitiendo la supervisión y el control remoto de las instalaciones, el procesamiento de la información, la presentación de gráficos, la generación de informes, alarmas, almacenamiento de históricos, etc., con un bajo coste.

En este artículo se describe el diseño de un sistema SCADA adaptado para el control del acceso a la dieta en roedores de laboratorio de una manera automatizada, con la posibilidad para el usuario de programar diferentes horarios de acceso en función del procedimiento. Además, el usuario podrá recoger de la jaula de alojamiento las siguientes variables de control: temperatura, humedad, peso de los individuos y cantidad de dieta ingerida.

Los datos serán obtenidos de manera precisa y en tiempo real mediante diferentes sensores, pudiéndose supervisar de manera remota el estado de las instalaciones y de los animales mediante el uso de cámaras digitales. Datos e
En este artículo se describe el diseño de un sistema SCADA adaptado para el control del acceso a la dieta en roedores de laboratorio de una manera automatizada, con la posibilidad para el usuario de programar diferentes horarios de acceso en función del procedimiento. Además, el usuario podrá recoger de la jaula de alojamiento las siguientes variables de control: temperatura, humedad, peso de los individuos y cantidad de dieta ingerida imágenes se enviarán de forma inalámbrica hasta un controlador compacto, que albergará algoritmos de gestión de alimentación y protocolos de manejo y control, entre otros. La información se procesará en tiempo real y dará órdenes de control según las necesidades reales de los animales.

La fase preliminar del diseño ha consistido en la descripción del compartimento donde se aloja el animal y de los principales sensores empleados. Posteriormente, se ha realizado una simulación virtual para establecer los principales parámetros de monitorización y las limitaciones y avances sustanciales del prototipo. En fases posteriores se trabajará con un prototipo real y se propondrán una serie de mejoras del diseño inicial a partir de la experimentación en laboratorio. En este artículo se incluyen únicamente la fase preliminar y los resultados de la simulación. 


\section{Material y métodos}

A la hora del diseño de un equipo de estas características nos encontramos con varios retos. El primero de ellos es el coste. Los dispositivos comerciales actuales son caros, por lo que es difícil su uso a nivel general, limitándose este a momentos puntuales dentro del procedimiento experimental. Otro desafío es poder realizar un seguimiento del peso de los animales alojados en grupo. Con los dispositivos actuales, esto resulta difícil, ya que, con la mayoría de ellos, es necesario mantener a los animales de forma individualizada, lo que ocasiona variaciones en el bienestar animal que, a su vez, conducen a alteraciones en parámetros fisiológicos y de comportamiento. Este innovador sistema permitirá gestionar la dieta de forma eficiente con un control preciso de los aportes de alimento, minimizando los consumos y maximizando la producción, la calidad, la rentabilidad y el bienestar animal.

Teniendo en cuenta todos los aspectos descritos con anterioridad, se ha diseñado un prototipo inicial que incluye los elementos que se describen en el cuadro 1.

Cuadro 1. Elementos del prototipo de bajo coste adaptado para el control de la alimentación en roedores de laboratorio

\section{Elemento}

Alojamiento pequeño de roedores de laboratorio.

Célula de carga.

$$
\begin{aligned}
& \text { Célula de carga de escala portable (1 kg) } \\
& \text { y sensor de peso electrónico. Rango de } \\
& \text { suministro de energía: } 3,0 \mathrm{~V}-5,5 \mathrm{~V} \text {. }
\end{aligned}
$$

Sensor digital de temperatura.

Sensor de temperatura con encapsulación de acero inoxidable, resistente a la humedad y antioxidante. Rango de suministro de energía: 3,0 V-5,5 V.

Sensor digital de temperatura y humedad relativa.
Integra un sensor capacitivo de humedad y un termistor para medir el aire circundante. Señal digital en el pin de datos.
Función

Alberga el animal e incluye los principales dispositivos de medida y control.

Monitorización del peso del animal.

Monitorización de la temperatura del alojamiento.

Monitorización de la humedad relativa del alojamiento.

Monitorización del consumo de comida y bebida del animal. cial de comida y bebida.
Incluye transductores de peso para medir alimentos y bebidas para roedores. Las dosis son definidas por el usuario. 
La idea principal es establecer un dispositivo compacto y portable que pueda integrarse en diferentes modelos de jaula. El prototipo que se ha desarrollado parte de un alojamiento de roedores de laboratorio convencional. Se trata de una jaula de laboratorio para roedor sin monitorización. Este tipo de jaula únicamente permite el alojamiento del animal sin ningún tipo de control adicional. A la jaula se le acoplan diferentes sensores (temperatura ambiente, humedad relativa, entre otros) para la monitorización de los animales y de las variables ambientales de una forma sencilla. Se controlará también la variación del peso o la cantidad de alimento consumida, aunque para este fin se debe diseñar un prototipo de jaula diferente y ello será objeto de futuros estudios.

\subsection{Sensores}

Para el control y la monitorización del sistema se van a emplear una serie de sensores que permitan el control del acceso a la dieta y la monitorización de las variables necesarias. Los sensores que se van a utilizar han sido descritos en el cuadro 1 y se pueden ver en la figura 1.

Figura 1. Prototipo con los principales sensores incluidos. Simulación virtual de los sensores para establecer la concepción del software y determinar los principales algoritmos de control

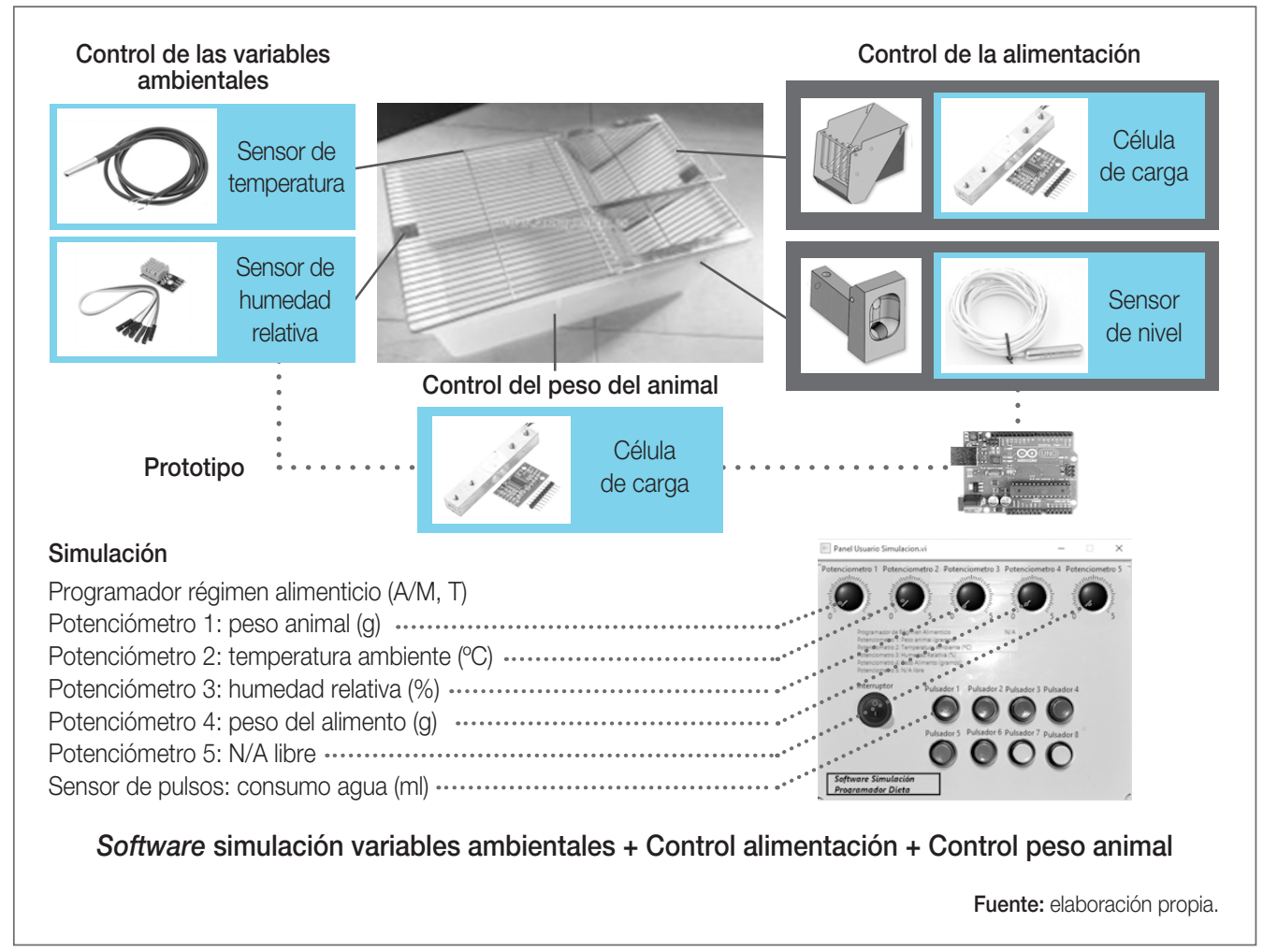


A continuación describimos cada uno de estos sensores:

- Célula de carga para monitorizar el peso del animal. En este caso se ha empleado una célula de carga con salida analógica 4-20 mA con una precisión mínima de 12 bits. Este sensor se ha instalado en la jaula de alojamiento y el pesaje se ha hecho de manera automática. La célula de carga es de $1 \mathrm{~kg}$, ubicándose el sensor en la zona donde el animal se sitúa para hacer las ingestas. Así se puede pesar durante el proceso.

Para controlar la ingesta de comida y bebida, dependiendo del tipo de jaula, se han instalado los dos tipos de sensores que se citan a continuación. Estos sensores recogen la información necesaria para conocer la cantidad de alimentos (g) ingeridos y la cantidad de agua ( $\mathrm{ml})$.

- Célula de carga para monitorizar el peso del alimento (500 g). De igual modo, para el control del consumo de alimento se ha integrado una solución mediante una célula de carga que está continuamente pesando el recipiente donde se deposita el alimento.

- Sensor de nivel. Para controlar el consumo de agua, se ha integrado una solución mediante una sonda de nivel. Los pulsos se detectan en el software directamente. Adicionalmente, en la jaula se han instalado sensores de control del ambiente con el fin de controlar las diferentes variables (temperatura, humedad relativa, entre otras) dentro de los valores que marcan las directrices europea y nacional (Directiva 2010 63/UE, de 22 de septiembre, y Real Decreto 53/2013, de 1 de febrero).

- Sensor de temperatura para controlar las variables climáticas del entorno. Se ha instalado un sensor en la parte superior del recipiente donde se encuentra alojado el animal.

- Sensor de humedad para controlar las variables climáticas del entorno. Se ha instalado un sensor en la parte superior del recipiente donde está alojado el animal.

Todos los sensores se han instalado en zonas de la jaula donde los animales no pueden acceder al cableado con la finalidad de evitar que estos deterioren los sensores.

En este primer prototipo no se han incluido cámaras de control, pero los futuros modelos dispondrán de una cámara para complementar la información aportada por el resto de sensores.

\subsection{Software y comunicaciones}

Los sensores van directamente a las entradas de Arduino ${ }^{\circledR}$ para recibir la información en el software. Se trata de un hardware de bajo coste que permite integrar gran cantidad de algoritmos de control y gestionar muchos datos en tiempo real a un precio competitivo. 
En el prototipo ensayado, los datos obtenidos se envían de forma inalámbrica hasta un microcontrolador Arduino $\mathrm{UNO} \otimes$, que alberga algoritmos de gestión para el control de la alimentación y la supervisión de los diferentes parámetros de control del animal. La información de los sensores se procesa en el microcontrolador Arduino UNO ${ }^{\circledR}$ y, a su vez, se almacena en un ordenador personal por cable USB.

Para el prototipo desarrollado en las fases posteriores, se emplearán sistemas de comunicaciones diversos (wifi/GPRS/3G/4G o ESP8266 wifi, entre otros) que enviarán la información a un hosting externo donde se almacenarán los datos y se mostrarán ordenados, con interfaz de usuario amigable, accesible desde cualquier navegador web o aplicaciones móviles específicas, proporcionando los servicios asociados a cualquier SCADA (gestión de históricos, avisos y alarmas, cambios de consignas, supervisión de los animales e instalaciones, etc.), análogo al desarrollado por González-Esquiva et al. en 2017 en instalaciones de riego. Desde cualquier ordenador o dispositivo móvil se puede acceder a través de aplicaciones específicas, capaces de interactuar con el controlador compacto, cambiar consignas, visualizar históricos, ver datos en tiempo real, etc. El sistema enlazará hardware y software y permitirá obtener datos específicos para usos diversos.

En la concepción del software se contempla también que, en determinadas circunstancias, los sensores no puedan medir debido a las características del sistema de alojamiento empleado. Para ello, en el software, se ha incorporado un control manual. Con este control manual, el operario especifica la cantidad de agua y comida que deposita para un tiempo determinado, conociendo así el consumo hasta su siguiente reposición manual.

\subsection{Descripción del software}

Inicialmente, para recoger toda la información de los sensores y mostrarla de forma amigable, se ha empleado el software de programación gráfica LabVIEW®. Mediante este software se programa un régimen horario, dependiendo de las consignas que dicta un operario experto, para cada recipiente individualmente.

Se ha creado un clúster de recipientes en los cuales se puede programar de forma independiente el tipo de régimen alimenticio que tendrá esa unidad de alojamiento durante un tiempo determinado. Mientras se lleve a cabo este régimen alimenticio, se estará monitorizando el peso del animal, así como la temperatura y la humedad ambiente para comprobar cómo afecta. El software planteado permite el control de un número ilimitado de alojamientos con la salvedad de ampliar el número de microcontroladores necesarios.

En cuanto al suministro de la dieta, los regímenes de alimentación que contempla el software se detallan a continuación:

- Alimentación AL. Este régimen alimenticio supone el libre acceso al alimento durante las 24 horas del día, pero controlando otra serie de parámetros, como 
las condiciones ambientales y la cantidad de alimento ingerida por los animales durante un periodo de tiempo predeterminado.

- Alimentación restringida (AR). Este tipo de régimen implica una restricción dietética moderada con objeto de limitar la sobrealimentación sin que se produzcan deficiencias en algún nutriente o una desnutrición del animal. Dentro de este tipo de alimentación hay diferentes modalidades. El grupo de San Antonio, del National Institute on Aging (NIA), desarrolla diferentes sistemas de AR para un amplio grupo de animales, incluidos primates, analizando exhaustivamente sus ventajas e inconvenientes, y se han incluido en este software.

- AR moderada. Se restringe en torno al $30 \%$ de la ingesta diaria, desde las seis a las ocho semanas de edad (unos 12-14 g frente a los 17-20 g/día en $A L$, para rata).

- AR estricta. El animal recibe únicamente entre un 50 y un $60 \%$ del consumo máximo AL. Mediante este método se han mantenido ratas hasta los 36-40 meses de edad (fase senil), de incalculable valor para estudios relacionados con procesos degenerativos asociados al envejecimiento humano.

- Alimentación horaria o controlada. A los animales se les permite consumir tanta comida como quieran, pero solo durante periodos de tiempo determinados al día. Este régimen de alimentación se usa en experimentos en los cuales hay que estudiar parámetros fisiológicos o bioquímicos en respuesta a la ingesta y compararlos con los niveles de los mismos en fase de ayuno, o bien en aquellos que requieren un estado nutricional muy controlado. El empleo de sistemas automáticos de acceso de alimento es ideal para este tipo de régimen.

En los siguientes apartados se realiza una descripción del software específico desarrollado para el control de la alimentación y la bebida en roedores de laboratorio. A continuación, se detalla la simulación realizada con el software LabVIEW® en una maqueta virtual. Por último, se incluyen los resultados obtenidos con la simulación realizada.

\subsection{Software programador de dietas de roedores de laboratorio}

En cuanto al software propiamente dicho, se ha desarrollado una simulación del comportamiento de un alojamiento de roedores de laboratorio. Se habilita y deshabilita el acceso a la comida y se monitoriza el peso del animal, la cantidad de alimento y el agua ingerida. También se registran la temperatura ambiente y la humedad. El software ha sido desarrollado para experimentar una aplicación simulada de las variables en tiempo real que actúan sobre los animales y cómo se registraría para un caso real. El software es ampliable a un número ilimitado de jaulas para controlar el acceso individualmente a cada una de ellas, con su correspondiente registro de datos en formato Excel y gráficos de datos en tiempo real. 
Con el fin de ver las potencialidades del software citado previamente, se ha procedido a realizar una simulación mediante una maqueta virtual. En esta maqueta se sustituyen las señales de los sensores reales por pulsadores y potenciómetros virtuales. Esta opción es muy útil para realizar experimentos de funcionamiento del software sin necesidad de disponer del hardware (véase figura 1).

A partir de las señales generadas por la maqueta virtual, se programó un software de control mediante el programa LabWIEW®. A continuación, se detallan las principales pantallas del software y se describen las funciones de cada una de ellas.

\subsubsection{Inicio del software}

En esta pantalla se puede seleccionar si se quiere trabajar desde el hardware Arduino $\AA^{\text {y }}$ simular los diferentes sensores del prototipo mediante pulsadores y potenciómetros, o simular los valores directamente desde el software. Para entrar a la aplicación realizada, simplemente se debe pulsar sobre el botón Programador del régimen alimenticio (véase figura 2).

Figura 2. Pantalla principal del software para activar/desactivar la conexión del Arduino® y utilizar el software mediante simulación o maqueta física

\section{Programador \\ Régimen Alimenticio}

Programador de Régimen Alimenticio

Acceso Dieta Automático/Manual.

Acceso Dieta por Tiempo.

\section{Activar Arduino}

\section{Detener}

\section{Salir}

Fuente: elaboración propia. 


\subsubsection{Estado del sistema}

Esta pantalla muestra el estado del hardware y de los sensores instalados (véase figura 3). Se puede visualizar, entre otros, el estado de funcionamiento del microprocesador Arduino $\AA$, la señal y los datos de las comunicaciones y las entradas analógicas y digitales. Igualmente, se monitoriza el LED de estado, así como los valores actuales. Se contabiliza también el número de ciclos de iteración. Todo ello se visualiza en el ordenador personal donde están ejecutándose las diferentes simulaciones (tiempo, variación del peso del animal, consigna de alimentación, variación del consumo de alimento, variación del consumo de agua, entre otros). Para la simulación, aunque no se utilicen todas las conexiones disponibles del Arduino®, sí se han habilitado para futuras ampliaciones del software. En el software presentado se pueden encontrar ocho estados para entradas digitales y cinco estados para entradas analógicas. Para las entradas digitales, se utiliza solo una para la monitorización del consumo de agua $(\mathrm{ml})$ del animal. En cuanto a las entradas analógicas, se utilizan cuatro entradas para la monitorización del «peso animal», del «peso del alimento», de la «temperatura ambiente» y de la «humedad relativa».

Figura 3. Pantalla del estado del sistema

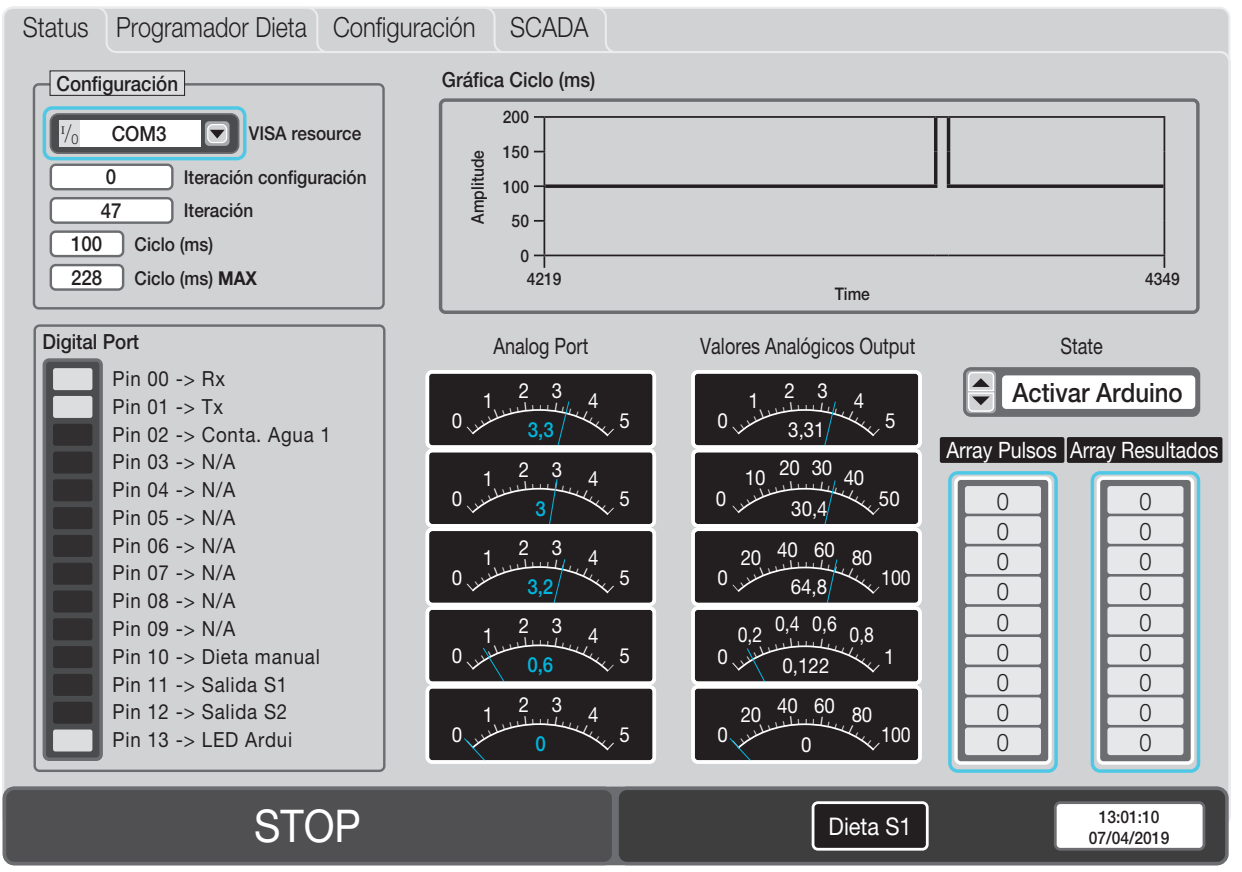

Fuente: elaboración propia. 


\subsubsection{Configuración}

Esta pantalla muestra el estado de las variables seleccionadas para el registro histórico de cada una de las jaulas experimentales. Desde el botón de Configuración se permite modificar el registro de cualquier variable del sistema. Esta configuración se guarda para ser usada en futuros experimentos, aunque se pueden realizar modificaciones según las necesidades del mismo. Cuando estas variables cambian, se genera un nuevo fichero Excel, ya que se modifica la cabecera del fichero. Se muestra también una aplicación sencilla donde se pueden visualizar los ficheros que hay registrados y abrirlos directamente desde el software (véase figura 4).

Figura 4. Pantalla de configuración de la programación del control de dieta, peso del animal y variables ambientales

\section{Status Programador Dieta Configuración SCADA \\ CONFIGURACIÓN HISTÓRICO DEL PROGRAMADOR DE ACCESO A DIETA}

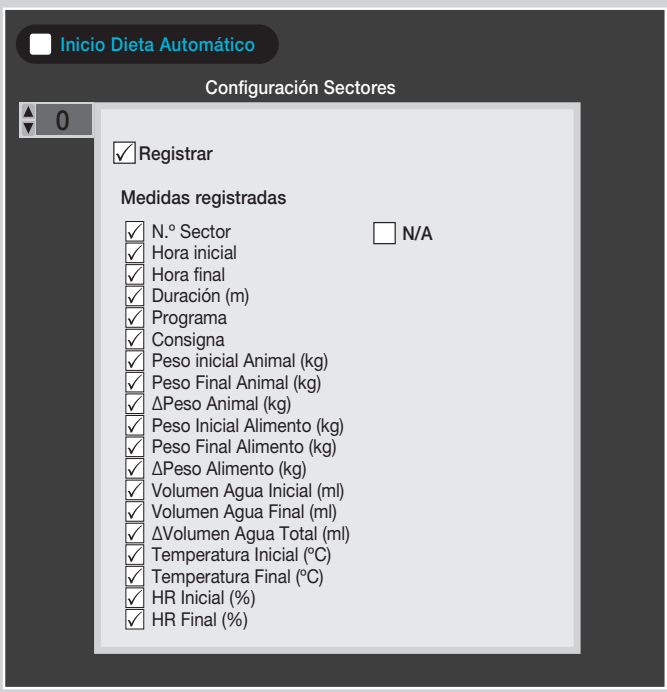

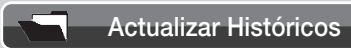

Lista Históricos

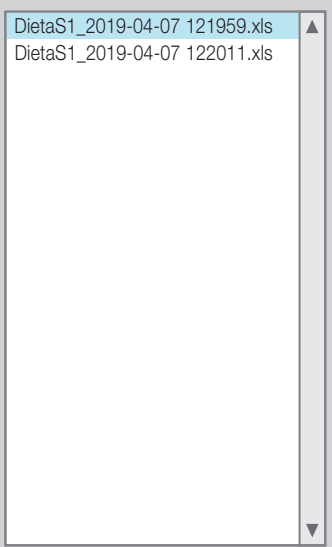

Cargar Históricos

\section{Configuración}

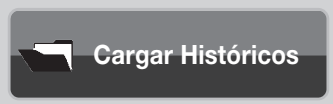

\section{STOP}




\subsubsection{Programador}

Esta pantalla muestra la programación del acceso a la dieta. A su vez se muestran las variables más representativas de la jaula seleccionada, monitorizándola en tiempo real (véase figura 5). Independientemente del programador, es posible realizar el acceso al alimento de forma manual, pulsando sobre Dieta manual cuando sea preciso. El programador de dieta está desarrollado para controlar el acceso a la dieta de dos formas: por tiempo (estableciendo diferentes tramos horarios en los que el animal tendrá acceso al comedero) o por gramos de comida consumidos (el acceso se cerrará una vez que se haya consumido cierta cantidad de dieta establecida previamente). A la programación asignada se le puede añadir una frecuencia de repetición de este programa; es decir, el usuario puede programar cuatro accesos de alimento para un día, cada 24 horas, de modo que se repetirá el mismo programa los días posteriores automáticamente. También permite guardar los programas y cargarlos en cualquier otro momento.

Figura 5. Pantalla del programador y mediciones en tiempo real

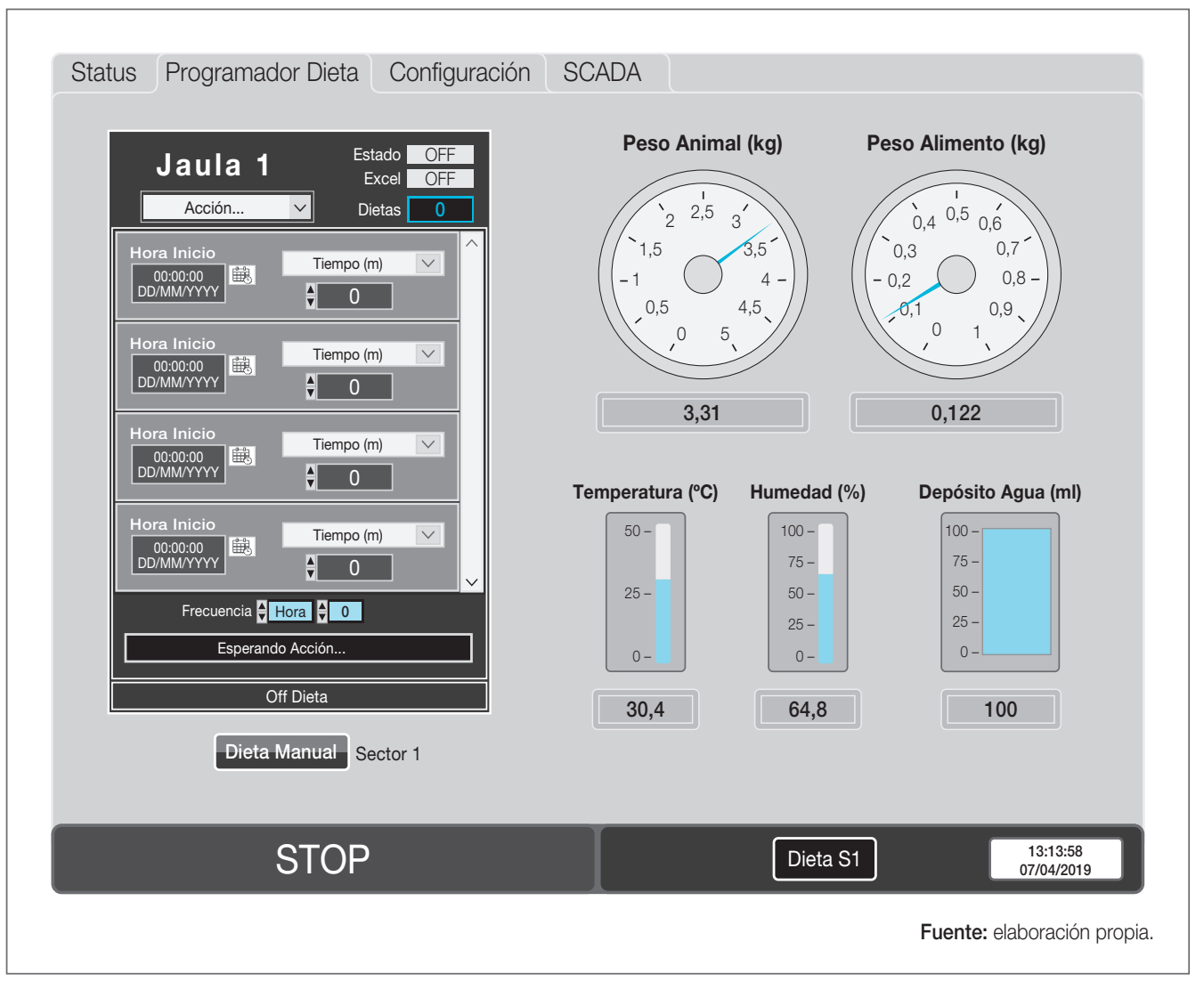




\subsubsection{Salidas formato Excel}

Las variables se registran en el fichero Excel con el siguiente formato para permitir al usuario trabajar con ellas de acuerdo a las necesidades del experimento (véase figura 6).

Figura 6. Salidas Excel

\begin{tabular}{|c|c|c|r|r|r|r|r|r|r|}
\hline & A & B & C & D & E & F & G & H & A \\
\hline $\mathbf{1}$ & Fecha & Hora & N. jaula & Hora inicial & Hora final & Duración $(\mathrm{m})$ & Programa & Consigna \\
\hline $\mathbf{2}$ & $07 / 04 / 2019$ & $12: 45: 35$ & Sector 1 & $12: 44: 35$ & $12: 45: 35$ & 1 & 0 & 1 \\
\hline $\mathbf{3}$ & & & & & & & & \\
\hline $\mathbf{4}$ & & & & & & & & \\
\hline
\end{tabular}

Fuente: elaboración propia.

\subsubsection{Salidas gráficas en tiempo real}

Facilita la interpretación de los datos, ofreciendo los gráficos correspondientes a la monitorización de las medidas, de las alarmas y del resto de información en tiempo real. El registro histórico de sus valores se realiza de manera automática. Los históricos de medidas, alarmas registradas y cambios de configuración pueden ser consultados como listados en pantalla.

\section{Resultados y discusión}

Para evaluar el software desarrollado, se realizaron varias simulaciones basándose en datos de instalaciones de alimentación de roedores que funcionan en la actualidad, cuyas características se resumen en el cuadro 2.

Cuadro 2. Regímenes de alimentación

\begin{tabular}{|c|c|c|c|c|}
\hline $\begin{array}{l}\text { Tipo de régimen } \\
\text { alimenticio }\end{array}$ & Horario & Usos & $\begin{array}{c}\text { Tipo de } \\
\text { alojamiento }\end{array}$ & $\begin{array}{l}\text { Parámetros } \\
\text { recogidos }\end{array}$ \\
\hline$A L$ & Acceso libre. & $\begin{array}{l}\text { No hay limitación en } \\
\text { el acceso al alimento. }\end{array}$ & Grupo. & $\begin{array}{l}\text { Parámetros ambien- } \\
\text { tales y cantidad total } \\
\text { de dieta ingerida. }\end{array}$ \\
\hline
\end{tabular}




\begin{tabular}{|c|c|c|c|c|}
\hline $\begin{array}{l}\text { Tipo de régimen } \\
\text { alimenticio }\end{array}$ & Horario & Usos & $\begin{array}{c}\text { Tipo de } \\
\text { alojamiento }\end{array}$ & $\begin{array}{l}\text { Parámetros } \\
\text { recogidos }\end{array}$ \\
\hline \multicolumn{5}{|l|}{$>$} \\
\hline $\begin{array}{l}\text { Horaria o contro- } \\
\text { lada }\end{array}$ & $\begin{array}{l}\text { Se suprime acceso } \\
\text { entre las } 02: 00 \mathrm{~h} \mathrm{y} \\
\text { las 11:00 h. }\end{array}$ & $\begin{array}{l}\text { Este régimen está indi- } \\
\text { cado para el estableci- } \\
\text { miento de periodos de } \\
\text { ayuno. }\end{array}$ & Grupo. & $\begin{array}{l}\text { Parámetros ambienta- } \\
\text { les y cantidad total de } \\
\text { dieta ingerida. }\end{array}$ \\
\hline Restringida & $\begin{array}{l}\text { Se impide el acce- } \\
\text { so a la dieta una vez } \\
\text { que el animal ha in- } \\
\text { gerido el } 70 \% \text { de la } \\
\text { cantidad habitual. }\end{array}$ & $\begin{array}{l}\text { Limita el acceso a la } \\
\text { dieta, útil en estudios } \\
\text { de envejecimiento para } \\
\text { reducir la ingesta duran- } \\
\text { te la vida del animal. }\end{array}$ & Individual. & $\begin{array}{l}\text { Parámetros ambien- } \\
\text { tales, peso del animal } \\
\text { y cantidad de dieta in- } \\
\text { gerida. }\end{array}$ \\
\hline
\end{tabular}

Fuente: elaboración propia.

Mediante el software se presentaron los valores medidos por los sensores del prototipo diseñado. Los valores estimados obtenidos durante la simulación para un periodo de 24 horas pueden ser fáciles de apreciar en las siguientes figuras. En la figura 7, se puede ver la evolución de los parámetros ambientales (humedad relativa [\%] y temperatura media $\left.\left[{ }^{\circ} \mathrm{C}\right]\right)$ durante 24 horas. En la figura 8 se puede observar la evolución del peso del animal (g) y la cantidad de dieta ingerida (g).

Figura 7. Datos ambientales (humedad relativa media [\%] y temperatura media $\left[{ }^{\circ} \mathrm{C}\right]$ ) obtenidos de la simulación

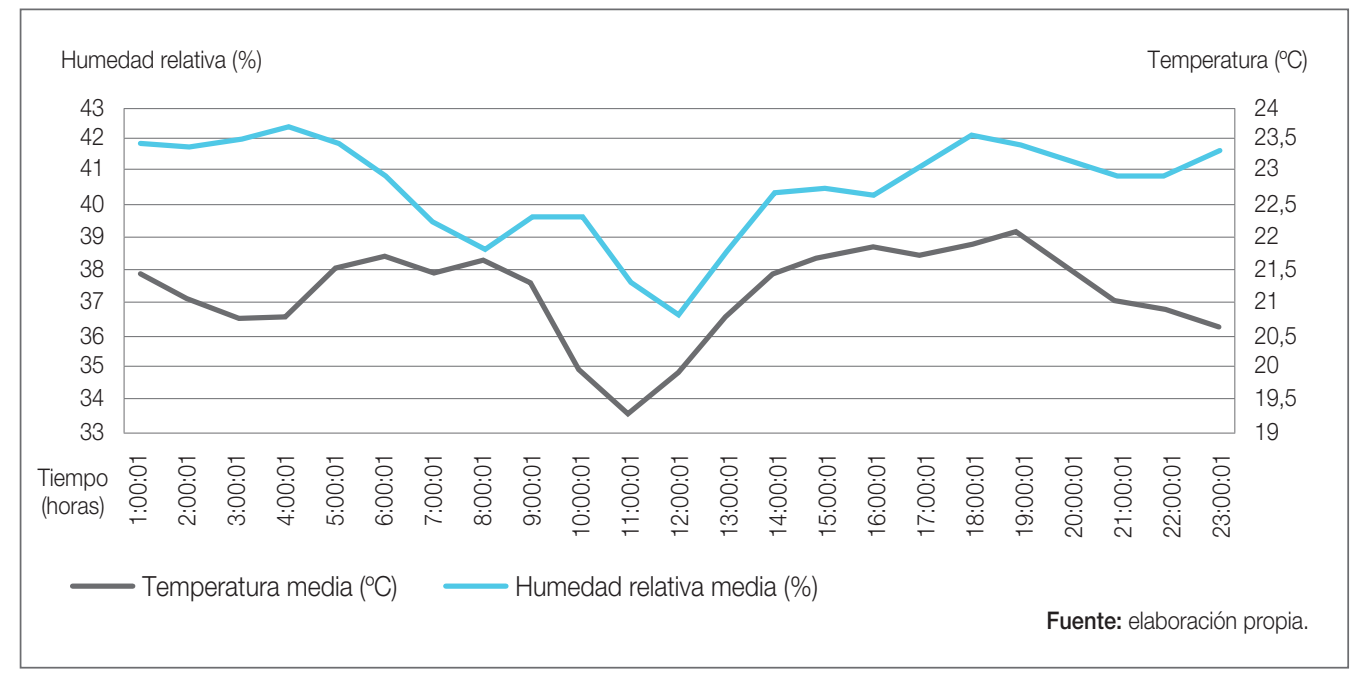


Figura 8. Evolución del peso del animal (g) y de la cantidad de dieta ingerida (g)

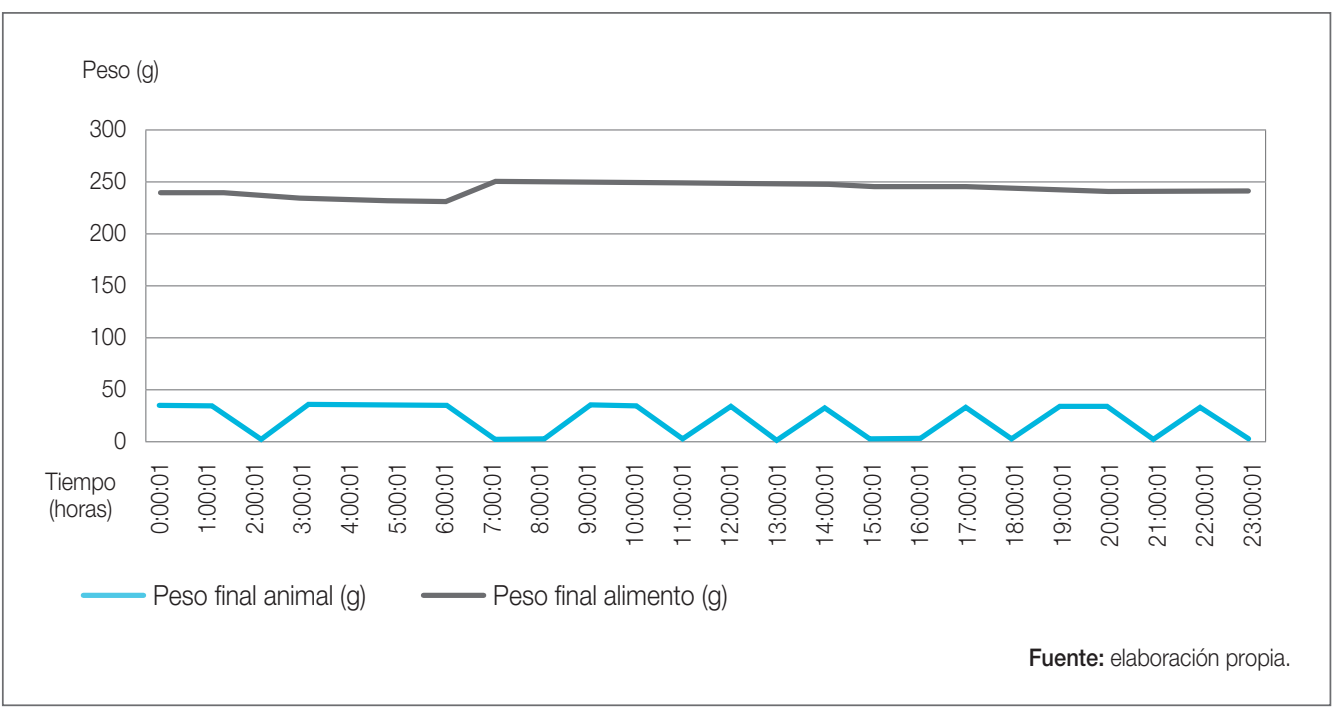

Los resultados observados son similares a los obtenidos de forma manual en una instalación real. En la figura 9 se muestra la variación del peso de un ratón C57/BL6 J de ocho semanas de edad durante 12 horas, con un pesado manual, y en la figura 10, la evolución de la temperatura y la humedad en una sala de la misma instalación.

Figura 9. Evolución del peso (g) de un ratón C57/BL6 $\mathrm{J}$ de ocho semanas de edad con pesado manual

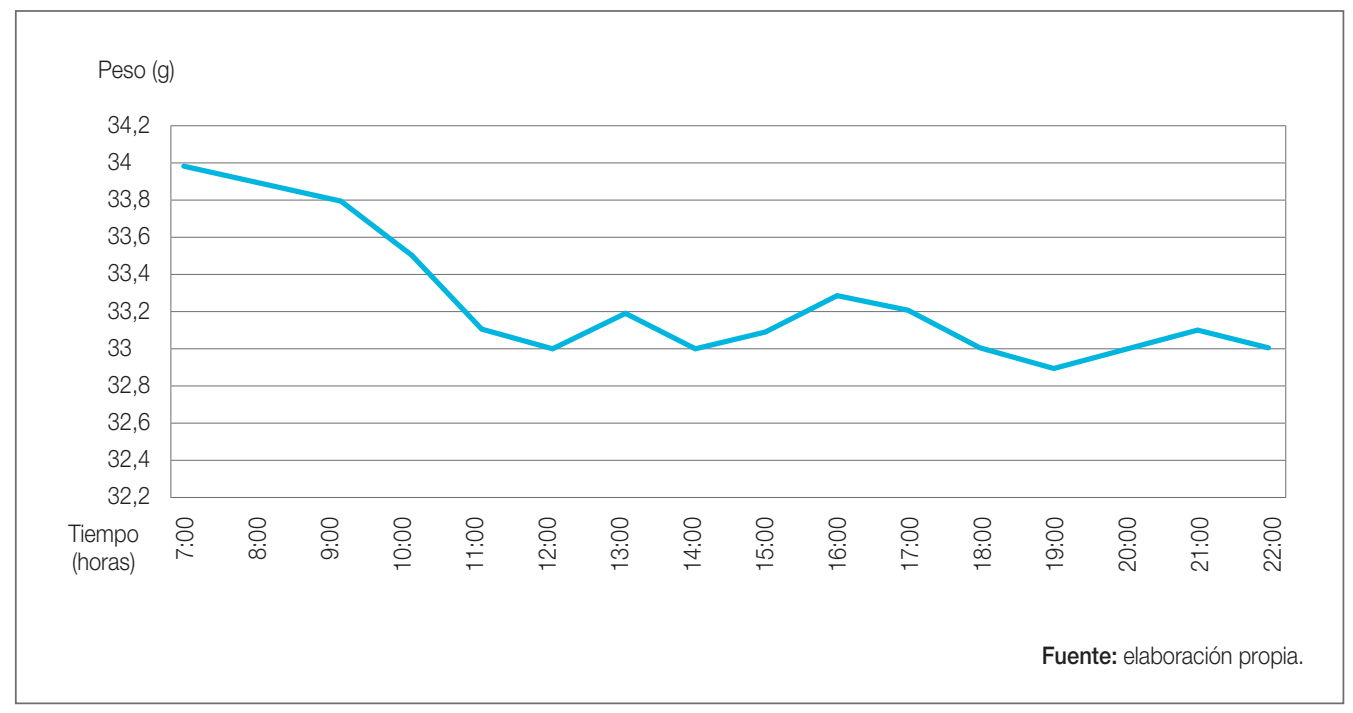


Figura 10. Evolución de los datos ambientales (humedad relativa media [\%] y temperatura media [ $\left.{ }^{\circ} \mathrm{C}\right]$ ) medidos manualmente en una de las salas de la misma instalación

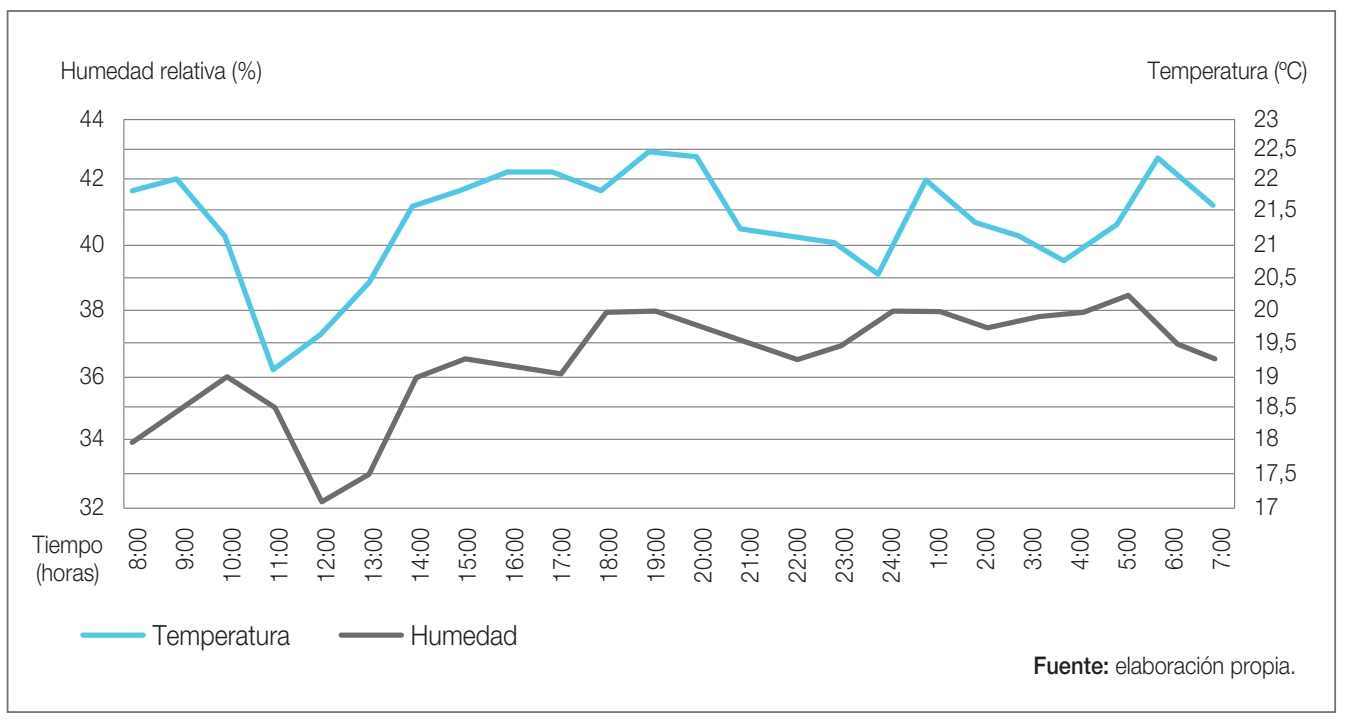

Estos resultados demostraron lo siguiente:

- Los valores ambientales simulados son similares a los reales.

- Si el alojamiento de roedores no tiene un sistema de seguimiento implementado, el software diseñado ayuda al operario a establecer los principales parámetros de control.

\section{Limitaciones del software}

El control de los alojamientos de roedores de laboratorio está influenciado por varios parámetros: temperatura ambiente, humedad relativa, control de la ingesta y control del peso. Aunque existen soluciones comerciales adecuadas, estas son caras y presentan limitaciones a la hora de implementar nuevas aplicaciones. Con la propuesta del software implementado y los sensores de bajo coste se abre la posibilidad de mejorar estos sistemas y adaptarlos a las necesidades específicas del laboratorio donde se emplee. Al implementar la aplicación del software presentado en este artículo:

- Se ha encontrado una solución alternativa a las de las casas comerciales existentes, que permite trabajar con un número ilimitado de alojamientos a un precio competitivo. 
- Los algoritmos empleados para el control ambiental permiten dar unos resultados similares a las instalaciones reales.

Actualmente se está desarrollando un experimento para comparar un prototipo real con una instalación convencional con el fin de establecer las limitaciones reales.

\section{Conclusiones}

Este artículo presenta una nueva aplicación de software, cuya función principal es controlar el acceso a la dieta y el peso del animal y la monitorización de diferentes parámetros ambientales (temperatura y humedad relativa). El dispositivo permite controlar los mismos parámetros que los equipos convencionales, pero con la ventaja de añadir prestaciones adicionales específicas a un precio muy competitivo. La aplicación ha sido desarrollada con un software para ordenador personal, pero se tiene previsto mejorar el sistema, ensayarlo en una instalación real y desarrollar módulos de comunicaciones y aplicaciones móviles de control. A partir de la experiencia realizada con el software de simulación se debería pasar a la fase de experimentación con un primer prototipo. Para esta fase se debería proceder a crear un alojamiento de roedores con

Este artículo presenta una nueva aplicación de software, cuya función principal es controlar el acceso a la dieta y el peso del animal y la monitorización de diferentes parámetros ambientales (temperatura y humedad relativa) las implementaciones descritas en esta publicación y ensayar los diferentes regímenes de alimentación, control del peso del animal, control de la comida y bebida, entre otros. Igualmente, se debería añadir el sistema de control mediante cámaras (no realizado hasta ahora) e incorporarlo al software. Otro aspecto a tener en cuenta en experimentaciones posteriores es el sistema de comunicaciones que permita recoger los datos y enviarlos a «la nube» para su gestión.

\section{Referencias bibliográficas}

Bains, R. S., Cater, H. L., Sillito, R. R., Chartsias, A., Sneddon, D., Concas, D., Keskivali-Bond, P., Lukins, T. C., Wells, S., Nolan, A. y Armstrong, P.M. (2016). Analysis of individual mou- se activity in group housed animals of different in bred strains using a novel automated home cage analysis system. Frontiers in Behavioral Neuroscience, 10, 106. 
Balcombe, J. P., Barnard, N. D. y Sandusky, C. (2004). Laboratory routines cause animal stress. Journal of the American Association for Laboratory Animal, 43(6), 42-51.

Bolaños, F., LeDue, J. M. y Murphy, T. H. (2017). Cost effective raspberry pi-based radio frequency identification tagging of mice suitable for automated in vivo imaging. Journal of Neuroscience Methods, 276, 79-83.

Carney, E. W., Zablotny, C. L., Marty, M. S., Crissman, J. W., Anderson, P., Woolhiser, M. y Holsapple, M. (2004). The effects of feed restriction during in utero and postnatal development in rats. Toxicological Science: An Official Journal of the Society of Toxicology, 82(1), 237-249.

Chacón, F., Esquifino, A. I., Perelló, M., Cardinali, D. P., Spinedi, E. y Álvarez, M. P. (2005). 24-hour changes in ACTH, corticosterone, growth hormone, and leptin levels in young male rats subjected to calorie restriction. Chronobiology International, 22(2), 253-265.

Duffy, P., Lewis, S. M., Mayhugh, M. A., Trotter, R. W., Thorn, B. T., Feuers, R. J. y Turturro, A. (2004). The effects of different levels of dietary restriction on non-neoplastic diseases in male Sprague-Dawley rats. Aging Clinical and Experimental Research Aging, 16(1), 68-78.

Duffy, P.H., Lewis, S. M., Mayhugh, M.A., Trotter, R. W., Latendresse, J. R., Thorn, B. T. y Feuers, R. J. (2004). The effects of different levels of dietary restriction on neoplastic pathology in the male Sprague-Dawley rat. Aging Clinical and Experimental Research, 16(6), 448-456.

Feuers, R. (1991). The relationship of dietary restriction to circadian variation in physiologic parameters and regulation of metabolism. Aging, 3(4), 399-401.

González-Esquiva, J. M., Oates, M. J., GarcíaMateos, G., Moros-Valle, B., Molina-Martínez,
J. M., Ruiz-Canales, A. (2017). Development of a visual monitoring system for water balance estimation of horticultural crops using low cost cameras. Computers and Electronics in Agriculture, 141, 15-26.

Goulding, E. H., Schenk, A. K., Juneja, P., MacKay, A. W., Wade, J. M. y Tecott, L. H. (2008). A robust automated system elucidates mouse home cage behavioral structure. Proceeding of the National Academy of Sciences of the United States of America, 105(52), 20.575-20.582.

Hubert, M., Laroque, P., Gillet, J. y Keenan, K. P. (2000). The effects of diet, ad libitum feeding, and moderate and severe dietary restriction on body weight, survival, clinical pathology parameters, and cause of death in control Sprague-Dawley rats. Toxicological Science: An Official Journal of the Society of Toxicology, 58, 195-207.

Keenan, K. P., Laroque, P. y Dixit, R. (1998). Need for dietary control by caloric restriction in rodent toxicology and carcinogenicity studies. Journal of Toxicology and Environmental Health, Part B, Critical Review, 1, 135-148.

Keenan, K. P., Laroque, P., Ballam, G. C., Soper, K. A., Dixit, R., Mattson, B. A., Adams, S. P. y Coleman, J. B. (1996). The effects of diet, ad libitum overfeeding, and moderate dietary restriction on the rodent bioassay: the uncontrolled variable in safety assessment. Toxicological Pathology, 24(6), 757-768.

Kemppinen, N., Meller A., Mauranen, K., Kohila, T. y Nevalainen, T. (2008). Work for food-A solution to restricting food intake in group housed rats? Scandinavian Journal of Laboratory Animal Science, 35(2), 81-90.

Leakey, J. E. A., Seng, J. E. y Allaben, W. T. (2003). Body weight considerations in the B6C3F1 mouse and the use of dietary control to standardize background tumor incidence in chronic bioassays. Toxicology and Applied Pharmacology, 193(2), 237-265. 
Masoro, E. J. (1995). Design issues in the use of the diet-restricted rodent model. En R. W. Hart, D. A. Neumann, R. T. Robertson (Eds.), Dietary Restriction: Implications for the Design and Interpretation of Toxicity and Carcinogenicity Studies (1. ${ }^{a}$ ed., pp. 41-50). Washington DC, EE: UU.: LSI Press.

Masoro, E. J. (2005). Overview of caloric restriction and ageing. Mechanisms of Ageing and Development, 126(9), 913-922.

Molina Martínez, J. M., Ruiz-Canales, A., Jiménez-Buendía, M., Soto, F. y García Fernández-Pacheco, D. (2014). SCADA platform combined with a scale model of trickle irrigation system for agricultural engineering education. Computer Applications in Engineering Education, 22(3).

Murphy, T. H., Boyd, J. D., Bolaños, F., Vanni, M. P., Silasi, G., Haupt, D. y Ledue, J. M. (2016). High-throughput automated home-cage mesoscopic functional imaging of mouse cortex. Nature Communicantions, 7.

Naoto I. (26 de junio de 2015). Development of balance for animals. A \& D Discover Precision. Recuperado de <http://www. aandd.jp/su pport/devstories/story35.html> (consultado el 29 de noviembre de 2019).

Pickering, R. G. y Pickering, C. E. (1984). The effects of reduced dietary intake upon the body and organ weights, and some clinical chemistry and haematological variates of the young Wistar rat. Toxicology Letters, 21(3), 271-277.

Richardson, C. A. (2015). The power of automated behavioural homecage technologies in characterizing disease progression in laboratory mice: a review. Applied Animal Behaviour Science, 163, 19-27.

Ritskes-Hoitinga, M. y Savenije, B. (2008). The power of moderate food restriction (Abs- tract). En C. Bernadi, M. R. Gamble (Eds.), Proceedings of the Tenth FELASA Symposium and the XIV ICLAS General Assembly \& Conference, 11-14 de junio de 2007, Cernobbio, Italia. Londres, Inglaterra, Reino Unido: Royal Society of Medicine Press.

Roe, F. J., Lee, P. N., Conybeare, G., Kelly, D., Matter, B., Prentice, D. y Tobin, G. (1995). The biosure study: influence of composition of diet and food consumption on longevity, degenerative diseases and neoplasia in wistar rats studied for up to 30 months post weaning. Food and Chemical Toxicology, 33(1), S1-S100.

Russell, W. M. S. y Burch, R. L. (1959). The Principles of Humane Experimental Technique (1. ${ }^{a}$ ed.). Londres, Inglaterra, Reino Unido: Methuen.

Scott, B. B., Brody, C. D. y Tank, D. W. (2013). Cellular resolution functional imaging inbehaving rats using voluntary head restraint. Neuron, 80(2), 371-384.

Snyder, D. L. y Towne, B. (1989). The effect of dietary restriction on serum hormone and blood chemistry changes in aging LobundWistar rats. Progress in Clinical and Biological Research, 287, 135-146.

Spiteri, N. J. (1982). Circadian patterning of feeding, drinking and activity during diurnal food access in rats. Physiology \& Behavior, 28(1), 139-147.

Turturro, A., Duffy, P. H. y Hart, R. W. (1993). Modulation of toxicity by diet and dietary macronutrient restriction. Mutation Research, 295(4-6), 151-164.

Ulman, E. A., Compton, D. y Kochanek, J. (2008). Measuring food and water intake in rats and mice. ALN Magazine, 12, 17-20 
Para los Grados en Derecho o en

Ciencias del Trabajo, Relaciones

Laborales y Recursos Humanos

- Administración y Finanzas

- Asistencia a la Dirección

- Secretariado

\section{Para los Grados en Administración y}

Dirección de Empresas o en Economía

- Administración y Finanzas

- Asistencia a la Dirección

- Comercio Internacional

- Gestión Comercial y Marketing

- Marketing y Publicidad

- Secretariado

- Transporte y Logística

\section{Para el Grado en Marketing}

- Administración y Finanzas

- Comercio Internacional

- Gestión Comercial y Marketing
- Gestión de Alojamientos Turísticos

- Gestión de Ventas y Espacios Comerciales

- Marketing y Publicidad

- Transporte y Logística

Para los Grados en Magisterio de Educación Infantil y Magisterio de Educación Primaria

- Animación de Actividades Físicas y Deportivas

- Educación Infantil

- Integración Social

- Animación Sociocultural y Turística

Para el Grado en Empresas y Actividades Turísticas

- Gestión Comercial y Marketing

- Gestión de Ventas y Espacios Comerciales

- Gestión de Alojamientos Turísticos

- Agencias de Viajes y Gestión de Eventos

- Guía, Información y Asistencias Turísticas

\footnotetext{
* De acuerdo a lo establecido en el Real Decreto 1618/2011, de 14 de noviembre, sobre reconocimiento de estudios en el ámbito de la educación superior.
} 


\section{Ingeniería de Tecnologías y Servicios de Telecomunicación}

El papel estratégico del sector de las TIC, y la aplicación creciente de estas en los distintos sectores de la sociedad, ha hecho aumentar la necesidad de profesionales de la telecomunicación, una demanda que crecerá exponencialmente en los próximos años. Este grado habilita para el ejercicio de la profesión de ingeniero técnico de telecomunicación, otorgando las competencias necesarias para conseguir las atribuciones profesionales de un ingeniero técnico de telecomunicación y ofreciendo una formación que capacita al estudiante a la hora de analizar, diseñar, implementar, explotar y gestionar sistemas, componentes y procesos del ámbito de las TIC.

\section{Psicología (Rama CC. de la Salud)}

Siguiendo el modelo científico-profesional de psicólogo (o scientist-practitioner), se trata de aportar a los alumnos los conocimientos científicos necesarios para comprender, interpretar, analizar y explicar el comportamiento humano, así como para evaluar e intervenir en el ámbito individual y social, con el fin de que los psicólogos y la psicología promuevan y mejoren la salud y la calidad de vida de las personas.

\section{Historia}

Se conjugan los conocimientos humanísticos básicos y generalistas con el aprendizaje de las herramientas y técnicas de las nuevas TIC. Los estudiantes adquirirán la formación, los conocimientos y las habilidades necesarias para permitirles el pleno desarrollo de las funciones relacionadas con la investigación y la enseñanza de la historia, con la finalidad de que comprendan y hagan comprensibles a los demás los acontecimientos del pasado.

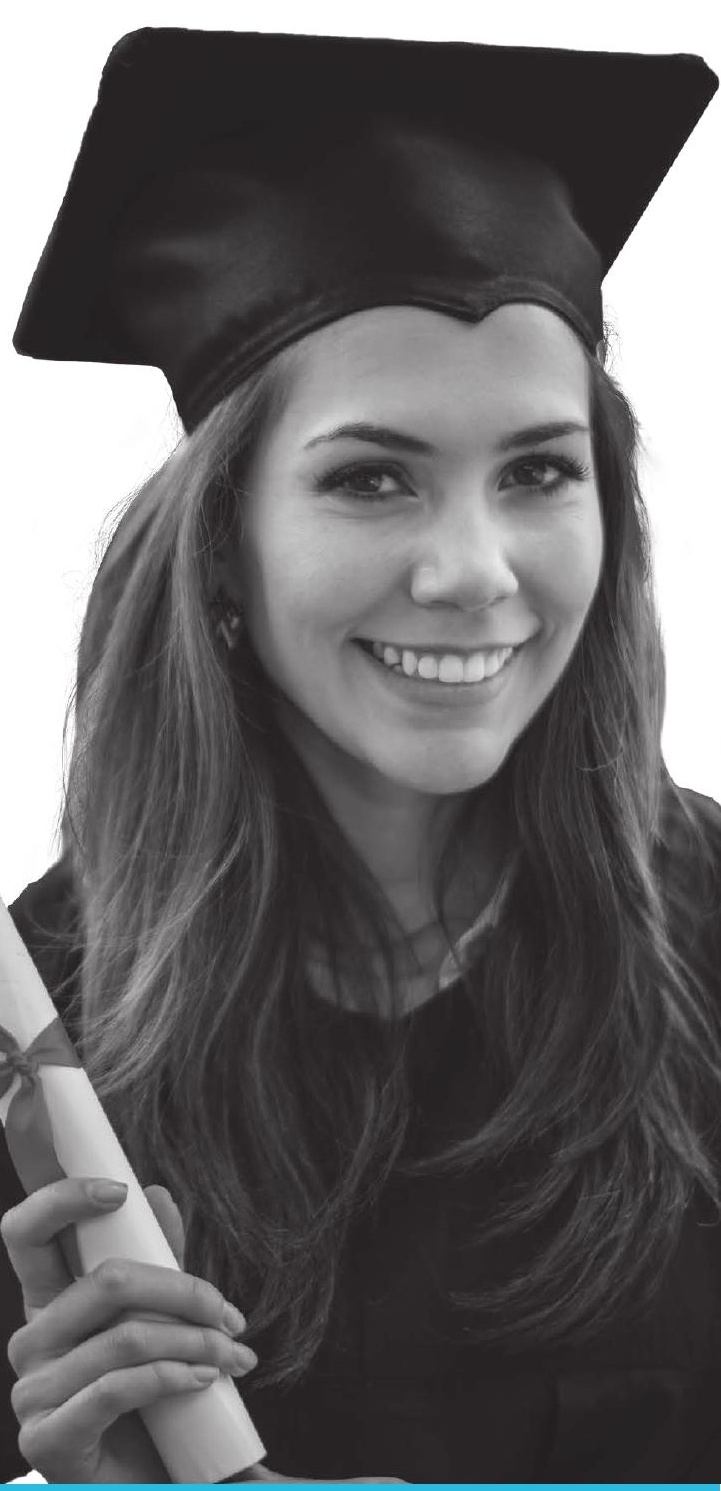

